\title{
High-Resolution Gadolinium-Enhanced MR Cisternography Using Compressed-Sensing T1 SPACE Technique for Detection of Intracranial CSF Leaks
}

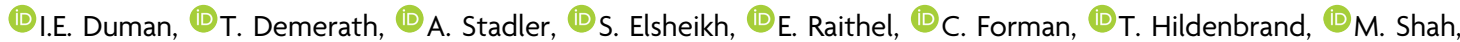 \\ D). Grauvogel, ${ }^{\circ}$ C. Scheiwe, ${ }^{-}$H. Urbach, and ${ }^{\circ}$ S. Meckel
}

\begin{abstract}
SUMMARY: In patients with CSF rhinorrhea, accurate identification of the CSF leakage site is crucial for surgical planning. We describe the application of a novel gadolinium-enhanced high-resolution 3D compressed-sensing TT SPACE technique for MR cisternography and compare findings with CT cisternography and intraoperative results. In our pilot experience with 7 patients, precise detection of CSF leaks was feasible using compressed-sensing TI SPACE, which appeared to be superior to CT cisternography.
\end{abstract}

ABBREVIATIONS: $\mathrm{CS}=$ compressed-sensing; $\mathrm{CTC}=\mathrm{CT}$ cisternography; MRC = MR cisternography; SPACE = sampling perfection with application-optimized contrasts by using different flip angle evolution

C

SF rhinorrhea develops after traumatic or iatrogenic injury to the dura mater, though spontaneous cases have also been reported. Traumatic CSF leaks often close spontaneously and may be treated conservatively. However, if persistent, the CSF leakage site may be a port of entry for bacteria, causing meningitis or other complications, and may need surgical repair. Identification of the precise preoperative leakage site is crucial for surgery and increases success rate while reducing the risk of complications. Although high-resolution CT is commonly the initial noninvasive study of choice, it may not be sufficient to accurately determine the location of a CSF leak. ${ }^{1,2}$ Intrathecal gadolinium-enhanced MR cisternography (MRC) has been recently introduced with studies reporting its safety and success in locating cranial CSF fistulas. ${ }^{1,2}$

Recently, a highly accelerated 3D compressed-sensing T1 sampling perfection with application-optimized contrasts by using different flip angle evolution (CS T1 SPACE) sequence (Siemens) has been introduced in MR neuroimaging, mainly as a tool for the assessment of intracranial vessel walls because of its excellent black-

Received May 19, 2020; accepted after revision August 16.

From the Departments of Neuroradiology (I.E.D., T.D., A.S., S.E., H.U., S.M.)

Otorhinolaryngology (T.H.), and Neurosurgery (M.S., J.G., C.S.), University of Freburg Medical Center, Faculty of Medicine, University of Freiburg, Freiburg Germany, and Siemens Healthcare GmbH (E.R., C.F.), Erlangen, Germany.

Paper previously presented at Virtual Conference of the German Society of Neuroradiology, October 7-9, 2020; Frankfurt, Germany.

Please address correspondence to Ikram Eda Duman, MD, Department of Neuroradiology, University of Freiburg Medical Center, Faculty of Medicine, University of Freiburg, Breisacherstr. 64, 79106 Freiburg, Germany; e-mail: edda ikram@hotmail.com

\footnotetext{
$\equiv$ Indicates article with supplemental on-line table.

$\Delta$ Indicates article with supplemental on-line photos.

DIndicates article with supplemental on-line videos.

http://dx.doi.org/10.3174/ajnr.A6852
}

blood imaging capabilities. ${ }^{3}$ The use of this technique for MRC after intrathecal gadolinium application may be advantageous because it offers a global 3D view of the entire skull base, meninges, and brain with high spatial detail and high SNR and contrast-to-noise ratio. Moreover, fusion images combining 3D CS T1 SPACE with highresolution CT may add important information on bony landmarks for surgical planning and intraoperative navigation.

This article illustrates our pilot experience using high-resolution CS T1 SPACE MRC for presurgical localization of CSF leaks in patients with CSF rhinorrhea. We compared imaging findings with CT cisternography (CTC) and intraoperative results.

\section{TECHNIQUES}

After introduction of a novel CS T1 SPACE MRC technique, we perform combined CTC and MRC in all patients with CSF rhinorrhea confirmed by positive B2-transferrin test result. The indication for cisternography was given by a neurosurgeon or otorhinolaryngologist based on the patient's clinical findings suspicious of a cranial CSF leak. Written informed consent for the intrathecal gadolinium injection as an off-label application and for the MR imaging scan was obtained from all patients.

After intrathecal injection of iodine contrast agent $(15 \mathrm{~mL}$ of Solutrast $250 \mathrm{M}$, Bracco Altana Pharma), saline $(4 \mathrm{~mL})$ mixed with $0.5 \mathrm{~mL}$ of gadoteridol (ProHance, Bracco Diagnostics) is injected into the subarachnoid space. Thereafter, patients are positioned prone in the $30^{\circ}-40^{\circ}$ Trendelenburg position for 10 minutes. CTC images are acquired first in a prone position. During transfer to the CT suite (10-20 $\mathrm{min}$ ), the patient is kept in bed in a prone position. Later (between 1.5-3 hours after contrast media injection), MRC is obtained in a supine position. Between both examinations, the patient is kept lying in bed in an observation room. 


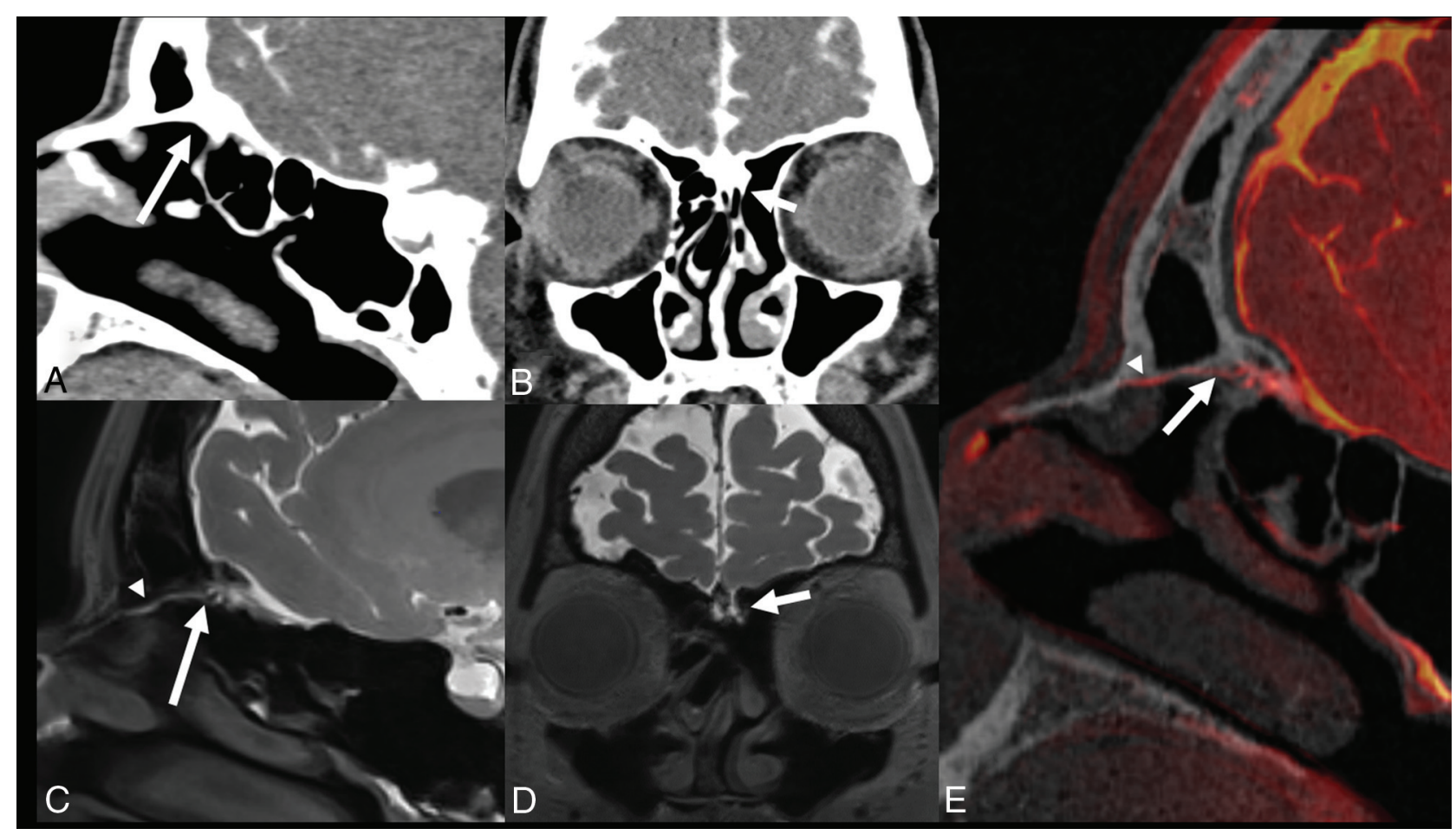

FIG 1. A 55-year-old woman (patient 1) who was readmitted for recurrent spontaneous left-sided CSF rhinorrhea and persistent slight headaches while sitting or standing after previous endoscopic sinus surgery. One year earlier, sealing of cribriform plate with TachoSil (Takeda Pharma) for suspected CSF leak had been performed. CT cisternography (soft tissue window level, W: 350; L: 50) is unremarkable, even in retrospect ( $A$ and $B$ arrows). CS SPACE MR cisternography demonstrates a subtle CSF leak originating from the anterior rim of the left cribriform plate near foramen cecum ( $C$ and $D$, arrows) with a thin CSF collection extending to the anterior nasal cavity (arrowheads in $C$ and E). The site correlated with the patient's intraoperative findings. After surgical repair, the patient was free of CSF rhinorrhea.

CTC is performed on a 64-section CT scanner (Somatom CT Definition AS, Siemens): section acquisition, $2 \times 0.6 \times 64$, by means of a z-flying focal spot; gantry rotation, 0.5 seconds; tube voltage, $80 \mathrm{kV}$; tube current-time product, $236 \mathrm{mAs} /$ pitch; sharp kernel (B60) reconstructed images (field of view, $100 \mathrm{~mm}$; section thickness, $0.6 \mathrm{~mm}$; increment, $0.4 \mathrm{~mm}$ ). Bone and soft tissue algorithms were applied to enhance bone and contrast media details.

For MRC, a highly accelerated CS T1 SPACE sequence was applied on a 3T whole-body MR scanner (Magnetom Prisma, Siemens) using a 64-channel head and neck coil. The sequence uses a Poisson-disc variable-density acquisition with elliptical $k$ space coverage. Image reconstruction was done by combination of CS with L1 norm-based regularization in the Wavelet domain and parallel imaging (number of iterations, 20; regularization parameter, 0.0013). A whole-head sagittal T1-weighted CS SPACE protocol (TR/TE, 800/5.1 ms; field of view, $210 \times 210 \mathrm{~mm}^{2}$; 256 slices; section thickness, $0.60 \mathrm{~mm}$; matrix, $384 \times 384$; fat saturation; pixel bandwidth, $450 \mathrm{~Hz} / \mathrm{px}$; echo-train length, 0 ; total scan time, 6:50 $\mathrm{min}$ ) with a $k$-space undersampling factor of 0.22 was implemented, which yields an approximate 5-fold acceleration compared with full $k$-space sampling. The CS SPACE T1 sequence had an almost isotropic $3 \mathrm{D}$ resolution $\left(0.5 \times 0.5 \times 0.6 \mathrm{~mm}^{3}\right){ }^{3}$

Syngo.via software (Siemens) was used to overlay 3D CS T1 SPACE and CTC images to delineate areas of leaks.

Between November 2019 and March 2020, 7 patients (mean age, 53.1 years; 5 females, 2 males) with CSF rhinorrhea underwent 8 combined CTC/MRC studies (On-line Table). All CSF leaks were precisely depicted on CS T1 SPACE images (Fig 1). In 5 CTC studies, leaks were missed (patient 1, Fig 1), falsely located (patient 2, study 1; On-line Fig $1 A-C$ ), or only suspected (patient 2, study 2; On-line Figs $1 D-F$, patients 3 and 4). All CSF leaks detected on MRC correlated with findings at surgical repair (On-line Videos 1 and 2).

\section{DISCUSSION}

In patients with CSF rhinorrhea, precise presurgical localization of CSF leaks is crucial for the success rate of surgery. High-resolution CT and CTC after intrathecal contrast application are both limited in the ability to demonstrate the site of a CSF leak, particularly in patients with multiple osseous defects or small or inactive leaks during imaging. ${ }^{1,2}$ Anatomically accurate delineation of even a very small dural dehiscence may also be very difficult to diagnose on CTC images in the absence of a larger bony defect. ${ }^{1,2}$

Standard gadolinium-enhanced MRC is a promising technique that may permit direct, sensitive visualization of the site of CSF leakage. ${ }^{1,2,4-6}$ Although intrathecal gadolinium injection is not approved by the US Food and Drug Administration, several studies have reported its safe off-label use with long-term follow-up after 4.2 years. ${ }^{1,2}$ Gadolinium-enhanced MRC has certain advantages over CTC. Although gadolinium distributes readily in the subarachnoid space, the high viscosity of the iodinated contrast agent may impair its free distribution and passage into slowly flowing CSF fistulas. Moreover, CTC 
requires high radiation dose and may not be able to differentiate CSF leakage from adjacent bone structures. In our series, all patients had confirmation of CSF leakage by surgery. In 5 of 8 CTC studies, the exact site of leakage could not be identified or was only suspected, and in 1 case, the suspected bony defect did not correlate with the site of leakage (patient 4). Related to different patient positioning in MR imaging (supine) and CT (prone), whereas more posterior dependent accumulation of leaked fluid was visible on MRC, CT showed anterior accumulation toward the nasal cavity (patient 4).

The application of 3D CS T1 SPACE for MRC appears particularly attractive because it covers the entire skull base and sinonasal and brain structures. Precise visualization of CSF leaks is enhanced related to its almost isotropic submillimeter 3D resolution, high SNR and contrast-to-noise ratio, and increased contrast between bony or aerated air cell structures, CSF, and soft tissues. ${ }^{3}$ The conspicuity of contrast medium leakage may also be enhanced because of saturation of medullary bone fat in the cranial base. Compared with earlier reports of gadolinium-enhanced MRC techniques that used spin-echo T1-weighted imaging at 1.5 $\mathrm{T},{ }^{4,5}$ the CS SPACE T1 technique appears advantageous for visualization of small CSF leaks because of 3D capability, increased spatial resolution, and SNR at 3T.

However, delineation of osseous anatomy at the skull base remains indispensable for surgical planning and intraoperative navigation. Thus, MRC-CTC fusion images combine the advantages of both techniques and allowed, in our series, accurate localization of the CSF leaks. Such fusion images may be alternatively created by combining MRC with low-dose bone-window CT images, thereby skipping the necessity for contrast-enhanced CTC.

Shortcomings of this study relate to the small number of retrospective cases, representing our very early clinical experience with the novel MRC method, which has to our knowledge not yet been described before. Technical limitations relate to the relatively long CS data processing times and relative vulnerability of the sequence to motion.

\section{CONCLUSIONS}

High-resolution gadolinium-enhanced CS T1 SPACE MRC is a promising method for detection of CSF leaks in patients with CSF rhinorrhea. In our pilot experience, this technique appears superior to standard CTC. Future prospective comparative studies are necessary to define the diagnostic accuracy of this technique.

Disclosures: Samer Elsheikh-UNRELATED: Grants/Grants Pending: Bracco Imaging*; Payment for Lectures Including Service on Speakers Bureaus: Daiichi Medical. Esther Raithel-UNRELATED: Employment: Siemens Healthcare GmbH, Comments: I am an employee. Christoph Forman-RELATED: Other: Employee of Siemens Healthcare GmbH, Comments: Salary; UNRELATED: Employment: Siemens Healthcare $\mathrm{GmbH}$; Stock/Stock Options: Siemens Healthineers AG. Horst Urbach—UNRELATED: Board Membership: Co-editor Clin Neuroradiol; Payment for Lectures Including Service on Speakers Bureaus: Bayer, Bracco, Eisai, Stryker, UCB pharma. Stephan Meckel—UNRELATED: Grants/Grants Pending: Bracco S.p.A., Italy.* *Money paid to institution.

\section{REFERENCES}

1. Reddy M, Baugnon K. Imaging of cerebrospinal fluid rhinorrhea and otorrhea. Radiol Clin North Am 2017;55:167-87 CrossRef Medline

2. Eljazzar R, Loewenstern J, Dai JB, et al. Detection of cerebrospinal fluid leaks: is there a radiologic standard of care? A systematic review. World Neurosurg 2019;127:307-15 CrossRef Medline

3. Guggenberger K, Krafft AJ, Ludwig U, et al. High-resolution compressed-sensing T1 black-blood MRI: a new multipurpose sequence in vascular neuroimaging? Clin Neuroradiol 2019 Dec 18 [Epub ahead of print] CrossRef Medline

4. Aydin K, Terzibasioglu E, Sencer S, et al. Localization of cerebrospinal fluid leaks by gadolinium-enhanced magnetic resonance cisternography: a 5-year single-center experience. Neurosurgery 2008;62: 584-89 CrossRef Medline

5. Selcuk H, Albayram S, Ozer H, et al. Intrathecal gadoliniumenhanced MR cisternography in the evaluation of CSF leakage. AJNR Am J Neuroradiol 2010;31:71-75 CrossRefMedline

6. Jinkins JR, Rudwan M, Krumina G, et al. Intrathecal gadoliniumenhanced MR cisternography in the evaluation of clinically suspected cerebrospinal fluid rhinorrhea in humans: early experience. Radiology 2002;222:555-59 CrossRef Medline 